\title{
Agradecimento aos Revisores da Revista Brasileira de Cardiologia Invasiva - 2008
}

A

Revista Brasileira de Cardiologia Invasiva agradece aos membros do Conselho Editorial e aos revisores ad hoc abaixo listados, responsáveis pela revisão dos trabalhos publicados no ano de 2008, que possibilitaram a publicação de artigos de alto nível científico, dentro do mais absoluto rigor ético:

Alexandre Azmus

Instituto de Cardiologia do Rio Grande do Sul, Fundação Universitária de Cardiologia

Porto Alegre, RS

Alexandre Schaan de Quadros

Instituto de Cardiologia do Rio Grande do Sul,

Fundação Universitária de Cardiologia

Porto Alegre, RS

André Labrunie

Hospital do Coração

Londrina, PR

Carlos Augusto Cardoso Pedra

Instituto Dante Pazzanese de Cardiologia;

Hospital do Coração - Associação do Sanatório Sírio

São Paulo, SP

Claudia Jacob Chaves

Tufts University School of Medicine

Boston, MA, Estados Unidos

Claudia Maria Rodrigues Alves

Disciplina de Cardiologia - UNIFESP-EPM

São Paulo, SP

Cristiano de Oliveira Cardoso

Instituto de Cardiologia do Rio Grande do Sul,

Fundação Universitária de Cardiologia

Porto Alegre, RS

Edson Antonio Bregagnollo

Faculdade de Medicina de Botucatu - UNESP

Botucatu, SP

Eduardo Missel

Hospital Ecoville

Curitiba, PR

Expedito Eustáquio Ribeiro da Silva

Instituto do Coração do Hospital das Clínicas da

Faculdade de Medicina da USP

São Paulo, SP

Fábio Sândoli de Brito Jr.

Hospital Israelita Albert Einstein

São Paulo, SP

Fernando Stuchi Devito

Hospital São Domingos;

Hospital Padre Albino

Catanduva, SP

Francisco José Araújo Chamié Queiroz

Setor de Hemodinâmica Pediátrica do Hospital dos Servidores do Estado - MS-RJ

Rio de Janeiro, RJ
Gilberto Lahorgue Nunes

Hospital São Francisco

Porto Alegre, RS

Hélio Roque Figueira

Clínica São Vicente;

Hospital CardioTrauma Ipanema

Rio de Janeiro, RJ

José Antonio Marin-Neto

Hospital das Clínicas -

Faculdade de Medicina da USP

Ribeirão Preto, SP

José de Ribamar Costa Junior

Instituto Dante Pazzanese de Cardiologia

São Paulo, SP

Luiz Alberto Mattos

Instituto Dante Pazzanese de Cardiologia

São Paulo, SP

Marinella Centemero

Instituto Dante Pazzanese de Cardiologia

São Paulo, SP

Maurício Rezende Barbosa

Centro de Treinamento em Hemodinâmica -

Hospital Biocor

Belo Horizonte, MG

Paulo Ricardo Avancini Caramori

Hospital São Lucas, PUCRS.

Hospital Mãe de Deus

Porto Alegre, RS

Pedro Alves Lemos Neto

Instituto do Coração do Hospital das Clínicas -

Faculdade de Medicina da USP

São Paulo, SP

Ricardo Alves da Costa

Instituto Dante Pazzanese de Cardiologia

São Paulo, SP

Rodolfo Staico

Instituto Dante Pazzanese de Cardiologia

São Paulo, SP

Rogério Sarmento-Leite

Instituto de Cardiologia do Rio Grande do Sul,

Fundação Universitária de Cardiologia

Porto Alegre, RS

Valmir Fernandes Fontes

Instituto Dante Pazzanese de Cardiologia

São Paulo, SP 